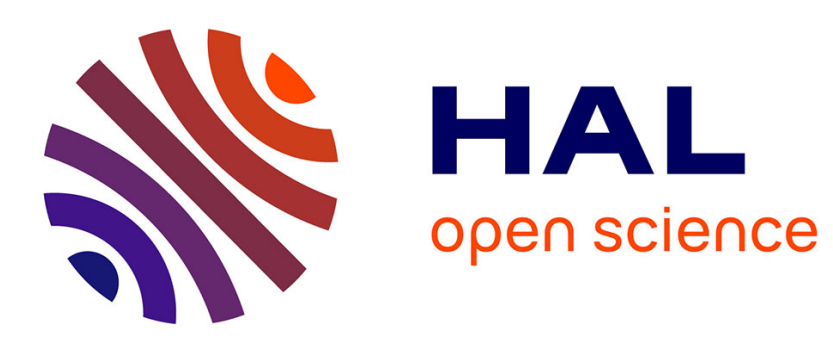

\title{
On the Möbius function of the locally finite poset associated with a numerical semigroup
}

Jonathan Chappelon, Jorge Luis Ramírez Alfonsín

\section{To cite this version:}

Jonathan Chappelon, Jorge Luis Ramírez Alfonsín. On the Möbius function of the locally finite poset associated with a numerical semigroup. Semigroup Forum, 2013, 87 (2), pp.313-330. 10.1007/s00233012-9458-3 . hal-00730229v3

\section{HAL Id: hal-00730229 \\ https://hal.science/hal-00730229v3}

Submitted on 30 Mar 2016

HAL is a multi-disciplinary open access archive for the deposit and dissemination of scientific research documents, whether they are published or not. The documents may come from teaching and research institutions in France or abroad, or from public or private research centers.
L'archive ouverte pluridisciplinaire HAL, est destinée au dépôt et à la diffusion de documents scientifiques de niveau recherche, publiés ou non, émanant des établissements d'enseignement et de recherche français ou étrangers, des laboratoires publics ou privés. 


\title{
ON THE MÖBIUS FUNCTION OF THE LOCALLY FINITE POSET ASSOCIATED WITH A NUMERICAL SEMIGROUP
}

\author{
JONATHAN CHAPPELON AND JORGE LUIS RAMÍREZ ALFONSÍN
}

\begin{abstract}
Let $S$ be a numerical semigroup and let $\left(\mathbb{Z}, \leq_{S}\right)$ be the (locally finite) poset induced by $S$ on the set of integers $\mathbb{Z}$ defined by $x \leq_{S} y$ if and only if $y-x \in S$ for all integers $x$ and $y$. In this paper, we investigate the Möbius function associated to $\left(\mathbb{Z}, \leq_{S}\right)$ when $S$ is an arithmetic semigroup.
\end{abstract}

Keywords: Möbius function, poset, numerical semigroup, arithmetic semigroup

MSC 2010: 20M15 ; 05A99 ; 06A07 ; 11A25 ; 20M05

\section{INTRODUCTION}

The Möbius function is an important concept associated to (locally finite) posets. Möbius function can be considered as a generalization of the classical Möbius arithmetic function on the integers (given by the Möbius function of the poset obtained from the positive integers partially ordered by the divisibility). Möbius function has been extremely useful to investigate many different problems. For instance, the inclusion-exclusion principle can be retrieved by considering the set of all subsets of a finite set partially ordered by inclusion. We refer the reader to [5] for a large number of applications of the Möbius function.

In this paper, we investigate the Möbius function associated to posets arising naturally from numerical semigroups as follows. Let $a_{1}, a_{2}, \ldots, a_{n}$ be $n \geq 1$ relatively prime positive integers and let $S=\left\langle a_{1}, a_{2}, \ldots, a_{n}\right\rangle$ denote the numerical semigroup generated by $a_{1}, a_{2}, \ldots, a_{n}$, that is,

$$
S=\left\langle a_{1}, a_{2}, \ldots, a_{n}\right\rangle=\left\{x_{1} a_{1}+x_{2} a_{2}+\cdots+x_{n} a_{n} \mid x_{1}, x_{2}, \ldots, x_{n} \in \mathbb{N}\right\} .
$$

Throughout this paper, we consider the structure of the poset induced by $S$ on the set of integers $\mathbb{Z}$, whose partial order $\leq_{S}$ is defined by

$$
x \leq_{S} y \quad \Longleftrightarrow \quad y-x \in S,
$$

for all integers $x$ and $y$. This (locally finite) poset will be denoted by $\left(\mathbb{Z}, \leq_{S}\right)$.

We denote by $\mu_{S}$ the Möbius function associated to $\left(\mathbb{Z}, \leq_{S}\right)$. As far as we are aware, the only known result concerning $\mu_{S}$ is an old theorem due to Deddens [1] that determines the value of $\mu_{S}$ when $S$ has exactly two generators. Here, we shall introduce and develop a new approach to investigate $\mu_{S}$ when $S$ is an arithmetic semigroup, that is, when $S=\langle a, a+d, \ldots, a+k d\rangle$ for some integers $a, d$ and $k \leq a-1$.

This is a self-contained paper and it is organized as follows. In the next section, we review some classic notions of the Möbius function and present some results needed for the rest of the paper. In Section 3, we give a new direct proof of Deddens' result, shorter than the original one (based on a recursive case-by-case analysis). In Section 4, we discuss results about arithmetic semigroups, in particular, we prove the existence of unique representations. The 
latter is a key result that will be used, in Section 5 , to give a recursive formula for $\mu_{S}$ when $S=\langle a, a+d, \ldots, a+k d\rangle$. Finally, in Section 6 , we propose an explicit formula for $\mu_{S}$ (based on the multiplicity function of a multiset) in the case when $k=2$ and $a$ is even.

Background information on numerical semigroups can be found in the books $[2,4]$.

\section{MöBius FUnCTION}

Let $(P, \leq)$ be a partially ordered set, or poset for short. The strict partial order $<_{P}$ is the reduction of $\leq_{P}$ given by, $a<_{P} b$ if and only if $a \leq_{P} b$ and $a \neq b$. For any $a$ and $b$ in the poset $P$, the segments between $a$ and $b$ are defined by

$$
\begin{array}{ll}
{[a, b]_{P}=\left\{c \in P \mid a \leq_{P} c \leq_{P} b\right\},} & ] a, b]_{P}=\left\{c \in P \mid a<_{P} c \leq_{P} b\right\}, \\
{\left[a, b\left[_{P}=\left\{c \in P \mid a \leq_{P} c<_{P} b\right\},\right.\right.} & ] a, b\left[_{P}=\left\{c \in P \mid a<_{P} c<_{P} b\right\} .\right.
\end{array}
$$

A poset is said to be locally finite if every segment has finite cardinality. In this paper, we only consider locally finite posets.

Let $a$ and $b$ be elements of the poset $P$. A chain of length $l \geq 0$ between $a$ and $b$ is a subset of $[a, b]_{P}$ containing $a$ and $b$, with cardinality $l+1$ and totally ordered by $<$, that is $\left\{a_{0}, a_{1}, \ldots, a_{l}\right\} \subset[a, b]_{P}$ such that

$$
a=a_{0}<_{P} a_{1}<_{P} a_{2}<_{P} \cdots<_{P} a_{l-1}<_{P} a_{l}=b .
$$

For any nonnegative integer $l$, we denote by $C_{l}(a, b)$ the set of all chains of length $l$ between $a$ and $b$. The cardinality of $C_{l}(a, b)$ is denoted by $c_{l}(a, b)$. This number is always finite because the poset $P$ is supposed to be locally finite. For instance, the number of chains $c_{2}(2,12)$, where the poset is the set $\mathbb{N}$ partially ordered by divisibility, is equal to 2 . Indeed, there are exactly 2 chains of length 2 between 2 and 12 in $[2,12]_{\mathbb{N}}=\{2,4,6,12\}$, which are $\{2,4,12\}$ and $\{2,6,12\}$.

For any locally finite poset $P$, the Möbius function $\mu_{P}$ is the integer-valued function on $P \times P$ defined by

$$
\mu_{P}(a, b)=\sum_{l \geq 0}(-1)^{l} c_{l}(a, b),
$$

for all elements $a$ and $b$ of the poset $P$. One can remark that this sum is always finite because, for $a$ and $b$ given, there exists a maximal length of a possible chain between $a$ and $b$ since the segment $[a, b]_{P}$ has finite cardinality.

The concept of Möbius function for a locally finite poset $(P, \leq)$ was introduced by Rota in [5] as the inverse of the zeta function in the incidence algebra of a locally finite poset. Let us see this with more detail. Consider the set $\mathcal{I}(P)$ of all real-valued functions $f: P \times P \longrightarrow \mathbb{R}$ for which $f(a, b)=0$ if $a \leq_{P} b$. The sum + and the multiplication by scalars . are defined as usual in $\mathcal{I}(P)$. The product of two functions $f$ and $g$ in $\mathcal{I}(P)$ is defined by

$$
(f \times g)(a, b)=\sum_{c \in[a, b]_{P}} f(a, c) g(c, b),
$$

for all $(a, b) \in P \times P$. Then $(\mathcal{I}(P),+, ., \times)$ appears as an associative algebra over $\mathbb{R}$. This is the incidence algebra of $P$. The Kronecker delta function $\delta \in \mathcal{I}(P)$, defined by

$$
\delta(a, b)=\left\{\begin{array}{cl}
1 & \text { if } a=b \\
0 & \text { otherwise } \\
2 &
\end{array}\right.
$$


for all $(a, b) \in P \times P$, is the identity element of $\mathcal{I}(P)$. The zeta function $\zeta_{P} \in \mathcal{I}(P)$ is defined by

$$
\zeta_{P}(a, b)= \begin{cases}1 & \text { if } a \leq_{P} b \\ 0 & \text { otherwise }\end{cases}
$$

for all $(a, b) \in P \times P$.

Rota [5] proved that the zeta function $\zeta_{P}$ (called the inverse function) is invertible in $\mathcal{I}(P)$ and showed that $\mu_{P}$ is recursively defined as follows: for all $(a, b) \in P \times P$, by

$$
\mu_{P}(a, a)=1 \quad \text { and } \quad \mu_{P}(a, b)=-\sum_{c \in\left[a, b\left[_{P}\right.\right.} \mu_{P}(a, c) \text { if } a<_{P} b .
$$

Let us see that both definitions of $\mu_{S}$ given by (1) and by (2) are equivalent. For, let $a$ and $b$ be two elements of the locally finite poset $P$ such that $a<_{P} b$. Then,

$$
c_{l}(a, b)=\sum_{c \in\left[a, b\left[\left[_{P}\right.\right.\right.} c_{l-1}(a, c)=\sum_{c \in] a, b]_{P}} c_{l-1}(c, b),
$$

for all positive integers $l$. Indeed, every chain $\left\{a_{0}, a_{1}, \ldots, a_{l}\right\} \in C_{l}(a, b)$ can be seen like an extension of a chain of $C_{l-1}\left(a, a_{l-1}\right)$ or of $C_{l-1}\left(a_{1}, b\right)$.

Obviously, the identity $\mu_{S}(a, a)=1$ directly comes from (1) since $c_{0}(a, a)=1$ and $c_{l}(a, a)=0$ for all $l \geq 1$. By combining (3) and (1), for all $a<_{P} b$, we obtain that

$$
\mu_{P}(a, b)=\sum_{l \geq 0}(-1)^{l} c_{l}(a, b)=c_{0}(a, b)+\sum_{l \geq 1}(-1)^{l} \sum_{c \in\left[a, b\left[_{P}\right.\right.} c_{l-1}(a, c) .
$$

Finally, since $a \neq b$, it follows that $c_{0}(a, b)=0$ and thus

$$
\mu_{P}(a, b)=\sum_{c \in\left[a, b\left[\left[_{P}\right.\right.\right.} \sum_{l \geq 0}(-1)^{l+1} c_{l}(a, c)=-\sum_{c \in\left[a, b\left[_{P}\right.\right.} \mu_{P}(a, c) .
$$

Similarly, using the second identity of (3), we can also prove that, whenever $a<_{P} b$, we have

$$
\mu_{P}(a, b)=-\sum_{c \in] a, b]_{P}} \mu_{P}(c, b)
$$

Therefore the two definitions of the Möbius function (for a locally finite posets) are the same. All the results presented in this paper are derived from the recursive formula presented in (2).

2.1. Poset of integers induced by a numerical semigroup. Let $S$ be a numerical semigroup and $\left(\mathbb{Z}, \leq_{S}\right)$ its associated poset. Observe that $\left(\mathbb{Z}, \leq_{S}\right)$ is a locally finite poset since $\left|[x, y]_{\left(\mathbb{Z}, \leq_{S}\right)}\right| \leq y-x$, for all $x, y \in \mathbb{Z}$. It is easy to see that $\mu_{S}$ can be considered as a univariable function of $\mathbb{Z}$. Indeed, for all $x, y \in \mathbb{Z}$ and for all $p \geq 0$, we have

$$
c_{l}(x, y)=c_{l}(0, y-x) .
$$

The above follows since the set $C_{l}(x, y)$ is in bijection with $C_{l}(0, y-x)$. Indeed the map that assigns the chain $\left\{x_{0}, x_{1}, \ldots, x_{l}\right\} \in C_{l}(x, y)$ to the chain $\left\{0, x_{1}-x_{0}, \ldots, x_{l}-x_{0}\right\} \in C_{l}(0, y-x)$ is clearly a bijection. Thus, by definition of $\mu_{S}$ and equality (4) we obtain

$$
\mu_{S}(x, y)=\mu_{S}(0, y-x)
$$

for all $x, y \in \mathbb{Z}$. 
In the sequel of this paper we shall only consider the reduced Möbius function $\mu_{S}: \mathbb{Z} \longrightarrow \mathbb{Z}$ defined by

$$
\mu_{S}(x)=\mu_{S}(0, x), \quad \text { for all } x \in \mathbb{Z} .
$$

This recursive formula given by (2) can be more easily presented when the locally finite poset is $\left(\mathbb{Z}, \leq_{S}\right)$.

Proposition 1. Let $S$ be a numerical semigroup and let $x \in \mathbb{Z} \backslash\{0\}$. Then,

$$
\mu_{S}(x)=-\sum_{y \in S \backslash\{0\}} \mu_{S}(x-y) \Longleftrightarrow \sum_{y \in S} \mu_{S}(x-y)=0 .
$$

Proof. From (2), we deduce that

$$
\mu_{S}(x)=-\sum_{y \in\left[0, x\left[\left[_{(\mathbb{Z}, \leq S}\right)\right.\right.} \mu_{S}(y)=-\sum_{\substack{y \in S \\ x-y \in S \backslash\{0\}}} \mu_{S}(y)=-\sum_{\substack{x-y \in S \\ y \in S \backslash\{0\}}} \mu_{S}(x-y) .
$$

The result follows since, by definition of $\mu_{S}, \mu_{S}(x-y)=0$ unless $x-y \in S$.

\section{DedDens' ReSUlt : NEW PROOF}

In [1], Deddens proved the following.

Theorem 1. [1] Let $a$ and $b$ be two relatively positive integers and let $S=\langle a, b\rangle$. Then, for all $x \in \mathbb{Z}$, we have

$$
\mu_{S}(x)=\left\{\begin{aligned}
1 & \text { if } x \geq 0 \text { and } x \equiv 0 \text { or } a+b \quad(\bmod a b) \\
-1 & \text { if } x \geq 0 \text { and } x \equiv a \text { or } b \quad(\bmod a b) \\
0 & \text { otherwise. }
\end{aligned}\right.
$$

Dedden's proof was based on a recursive argument and a case-by-case analysis. We may give the following direct proof of Theorem 1.

Proof of Theorem 1. We shall prove that

$$
\mu_{S}(x)=\mu_{S}(x-a b)
$$

for every $x \in \mathbb{Z} \backslash\{0, a, b, a+b\}$. The result then follows since $\mu_{S}(x)=0$ for all $x<0$, $\mu_{S}(0)=1, \mu_{S}(a)=\mu_{S}(b)=-1$ and $\mu_{S}(a+b)=c_{2}(0, a+b)-c_{1}(0, a+b)=2-1=1$.

Let us prove then equality (5). Let $S=\langle a, b\rangle=\left\{m_{a} a+m_{b} b \mid m_{a}, m_{b} \in \mathbb{N}\right\}$ and let $x \in$ $\mathbb{Z} \backslash\{0\}$. By Proposition 1 we already know that

$$
\mu_{S}(x)=-\sum_{y \in S \backslash\{0\}} \mu_{S}(x-y)=-\sum_{\substack{y \in S \backslash\{0\} \\ y-a \in S}} \mu_{S}(x-y)-\sum_{\substack{y \in S \backslash\{0\} \\ y-a \notin S}} \mu_{S}(x-y) .
$$

Since

$$
\sum_{\substack{y \in S \backslash\{0\} \\ y-a \in S}} \mu_{S}(x-y)=\sum_{z \in S} \mu_{S}((x-a)-z)=0, \quad \text { for } x-a \neq 0,
$$

then

$$
\mu_{S}(x)=-\sum_{\substack{y \in S \backslash\{0\} \\ y-a \notin S}} \mu_{S}(x-y), \quad \text { for } x \in \mathbb{Z} \backslash\{0, a\} .
$$


Moreover since $\{y \in S \backslash\{0\} \mid y-a \notin S\}=\left\{m_{b} b \mid m_{b} \in\{1,2, \ldots, a-1\}\right\}$ then

$$
\mu_{S}(x)=-\sum_{m_{b}=1}^{a-1} \mu_{S}\left(x-m_{b} b\right), \quad \text { for } x \in \mathbb{Z} \backslash\{0, a\} .
$$

By applying (6) for $x-b \in \mathbb{Z} \backslash\{0, a\}$, that is, $x \in \mathbb{Z} \backslash\{b, a+b\}$ we obtain that

$$
\mu_{S}(x-b)=-\sum_{m_{b}=2}^{a} \mu_{S}\left(x-m_{b} b\right), \quad \text { for } x-b \in \mathbb{Z} \backslash\{0, a\} .
$$

By combining (6) and (7), for $x \in \mathbb{Z} \backslash\{0, a, b, a+b\}$, we obtain that

$$
\begin{aligned}
\mu_{S}(x) & =-\sum_{m_{b}=1}^{a-1} \mu_{S}\left(x-m_{b} b\right)=-\mu_{S}(x-b)-\sum_{m_{b}=2}^{a-1} \mu_{S}\left(x-m_{b} b\right) \\
& =\sum_{m_{b}=2}^{a} \mu_{S}\left(x-m_{b} b\right)-\sum_{m_{b}=2}^{a-1} \mu_{S}\left(x-m_{b} b\right) \\
& =\mu_{S}(x-a b)
\end{aligned}
$$

as desired.

\section{Arithmetic Semigroups : PReliminary Results}

Let $S$ be a numerical semigroup. The Apéry set of $S$ with respect with $m \in S$ is defined as

$$
A p(S ; m)=\{x \in S \mid x-m \notin S\} .
$$

It is known that $A p(S ; m)$ constitutes a complete set a of residues $\bmod m$.

Roberts [3] has proved that if $S=\langle a, a+d, \ldots, a+k d\rangle$ with $\operatorname{gcd}(a, d)=1$ and $k \in\{1,2, \ldots, a-$ $1\}$ then

$$
A p(S ; a)=\left\{\left\lceil\frac{i}{k}\right\rceil a+i d \mid i \in\{0,1, \ldots, a-1\}\right\} .
$$

The following result gives a unique representation of elements in arithmetic semigroups.

Lemma 1. Let $x \in S=\langle a, a+d, \ldots, a+k d\rangle$ with $2 \leq k \leq a-1$. Then, there exists a unique triplet $\left(x_{0}, x_{i}, x_{k}\right) \in \mathbb{N} \times\{0,1\} \times\left\{0, \ldots,\left\lceil\frac{a}{k}\right\rceil\right\}$ such that

$$
x=x_{0} a+x_{i}(a+i d)+x_{k}(a+k d)
$$

for some $1 \leq i \leq k-1$ with $i x_{i}+k x_{k}<a$.

Proof. Let $x \in S$ and $x_{0}, \ldots, x_{k} \in \mathbb{N}$ such that

$$
x=x_{0} a+x_{1}(a+d)+x_{2}(a+2 d)+\cdots+x_{k}(a+k d) .
$$

Existence: Let $j_{1}$ and $j_{2}$ be two integers such that $0 \leq j_{1}, j_{2} \leq k$. We notice that $\left(a+j_{1} d\right)+$ $\left(a+j_{2} d\right)$ can be expressed as either

$$
\left(a+j_{1} d\right)+\left(a+j_{2} d\right)=a+\left(a+\left(j_{1}+j_{2}\right) d\right), \text { for } 0 \leq j_{1}+j_{2} \leq k
$$

or

$$
\left(a+j_{1} d\right)+\left(a+j_{2} d\right)=(a+k d)+\left(a+\left(j_{1}+j_{2}-k\right) d\right), \text { for } k \leq j_{1}+j_{2} \leq 2 k .
$$


So, by repeatedly adding consecutive terms from the expression of $x$ in (9), we obtain that there exists a triplet $\left(x_{0}, x_{i}, x_{k}\right)$ such that

$$
x=x_{0} a+x_{i}(a+i d)+x_{k}(a+k d),
$$

with $1 \leq i \leq k-1$ and $x_{i} \in\{0,1\}$. Moreover, we may suppose that $0 \leq x_{k} \leq\lfloor a / k\rfloor$. Otherwise, we use the following equality

$$
\begin{aligned}
\left(\left\lfloor\frac{a}{k}\right\rfloor+1\right)(a+k d) & =\left(\left\lfloor\frac{a}{k}\right\rfloor+1\right) a+\left(\left\lfloor\frac{a}{k}\right\rfloor k+k\right) d \\
& =\left(\left\lfloor\frac{a}{k}\right\rfloor+d+1\right) a+\left(\left\lfloor\frac{a}{k}\right\rfloor k+k-a\right) d \\
& =\left(\left\lfloor\frac{a}{k}\right\rfloor+d\right) a+\left(a+\left(\left\lfloor\frac{a}{k}\right\rfloor k+k-a\right) d\right),
\end{aligned}
$$

where $1 \leq\lfloor a / k\rfloor k+k-a \leq k$.

Finally, if $i x_{i}+k x_{k} \geq a$ then we consider the following representation

$$
\begin{aligned}
x & =x_{0} a+x_{i}(a+i d)+x_{k}(a+k d) \\
& =\left(x_{0}+x_{i}+x_{k}\right) a+\left(i x_{i}+k x_{k}\right) d \\
& =\left(x_{0}+x_{i}+x_{k}+d\right) a+\left(i x_{i}+k x_{k}-a\right) d \\
& =\left(x_{0}+x_{i}+x_{k}+d-1\right) a+\left(a+\left(i x_{i}+k x_{k}-a\right) d\right),
\end{aligned}
$$

where $0 \leq i x_{i}+k x_{k}-a \leq i x_{i}+k\lfloor a / k\rfloor-a \leq k-1+\lfloor a / k\rfloor k-a \leq k-1$. Obtaining the desired decomposition.

Uniqueness: Let us suppose that there exist two triplets of non-negative integers $\left(x_{0}, x_{i}, x_{k}\right)$ and $\left(y_{0}, y_{j}, y_{k}\right)$ such that

$$
x_{0} a+x_{i}(a+i d)+x_{k}(a+k d)=y_{0} a+y_{j}(a+j d)+y_{k}(a+k d),
$$

with $1 \leq i, j \leq k-1, x_{i}, y_{j} \in\{0,1\}, i x_{i}+k x_{k}<a$ and $j y_{j}+k y_{k}<a$. It follows that

$$
\left(i x_{i}+k x_{k}\right) d \equiv\left(j y_{j}+k y_{k}\right) d \quad(\bmod a),
$$

and since $\operatorname{gcd}(a, d)=1$ then

$$
i x_{i}+x_{k} k \equiv j y_{j}+k y_{k} \quad(\bmod a)
$$

Moreover, since $i x_{i}+k x_{k}<a$ and $j y_{j}+k y_{k}<a$, then

$$
i x_{i}+k x_{k}=j y_{j}+k y_{k} \text { or equivalently } i x_{i}-j y_{j}=k\left(y_{k}-x_{k}\right) .
$$

We have four cases.

Case 1) if $x_{i}=0$ and $y_{j}=1$ then $-j$ would be a multiple of $k$ which is impossible since $-1 \geq-j \geq-k$

Case 2) if $x_{i}=1$ and $y_{j}=0$ then $i$ would be a multiple of $k$ which is impossible since $1 \leq i \leq k$

Case 3) if $x_{i}=y_{j}=1$ then $i-j$ would be a multiple of $k$ but since $-k+2 \leq i-j \leq k-2$ then $i-j=0$ implying that $x_{k}=y_{k}$ and thus $x_{0}=y_{0}$.

Case 4) if $x_{i}=y_{j}=0$ then $k\left(x_{k}-y_{k}\right)=0$ and since $k \geq 1$ then $x_{k}=y_{k}$ and thus $x_{0}=y_{0}$. 
Let $\left(x_{0}, x_{i}, x_{k}\right) \in \mathbb{N} \times\{0,1\} \times\{0, \ldots,\lfloor a / k\rfloor\}$ with $1 \leq i \leq k-1$ and $i x_{i}+k x_{k}<a$. We shall denote by $\left[x_{0}, x_{i}, x_{k}\right]$ the element in $S$ given by the representation of Lemma 1 .

\section{RECURSIVE FORMULA}

We shall now present a recursive formula for $\mu_{S}$ when $S=\langle a, a+d, \ldots, a+k d\rangle$. The following key remark led us to guess such recursion. If $x=m_{a} a+m_{d} d$ such that $m_{a} \geq 0$ and $0 \leq m_{d} \leq a-1$ then,

$$
x \in S \quad \Longleftrightarrow \quad m_{a} \geq\left\lceil\frac{m_{d}}{k}\right\rceil .
$$

Theorem 2. Let $S=\langle a, a+d, \ldots, a+k d\rangle$ with $\operatorname{gcd}(a, d)=1$ and let $a=q k+r$ with $0 \leq r<k$. Let $x \in \mathbb{Z} \backslash\{0, a, a+k d, a+(a+k d)\}$, then

$$
\mu_{S}(x)= \begin{cases}\mu_{S}(x-q(a+k d))+\sum_{i=1}^{k-1} \mu_{S}(x-(a+i d)-q(a+k d)) & \\ -\mu_{S}(x-(a+i d)) & \text { if } r=0, \\ \mu_{S}(x-(q+1)(a+k d))+\sum_{i=r}^{k-1} \mu_{S}(x-(a+i d)-q(a+k d)) & \\ -\sum_{i=1}^{k-1} \mu_{S}(x-(a+i d)) & \text { if } r=1, \\ \mu_{S}(x-(q+1)(a+k d))+\sum_{i=1}^{r-1} \mu_{S}(x-(a+i d)-(q+1)(a+k d)) & \\ +\sum_{i=r}^{k-1} \mu_{S}(x-(a+i d)-q(a+k d))-\sum_{i=1}^{k-1} \mu_{S}(x-(a+i d)) & \text { if } r \geq 2 .\end{cases}
$$

Proof. Let $x \in \mathbb{Z} \backslash\{0\}$. As for the proof of Theorem 1, we have

$$
\begin{aligned}
\mu_{S}(x)= & -\sum_{y \in S \backslash\{0\}} \mu_{S}(x-y) \\
= & -\sum_{\substack{y \in S \backslash\{0\} \\
y-a \in S}} \mu_{S}(x-y)-\sum_{\substack{y \in S \backslash\{0\} \\
y-a \notin S}} \mu_{S}(x-y) \\
= & -\sum_{z \in S} \mu_{S}((x-a)-z)-\sum_{\substack{y \in S \backslash\{0\} \\
y-a \notin S}} \mu_{S}(x-y) \\
= & -\sum_{\substack{y \in S \backslash\{0\} \\
y-a \notin S}} \mu_{S}(x-y) \quad \text { if } \quad x-a \neq 0 .
\end{aligned}
$$

Let us now determine the set

$$
\{y \in S \backslash\{0\} \mid y-a \notin S\}=A p(S ; a) \backslash\{0\} .
$$

For, we consider the set $A p(S ; a)$ given by (8) in function of the unique representation of Lemma 1. We have three cases.

Case a) If $r=0$ then

$$
\begin{aligned}
& A p(S, a) \backslash\{0\}=\left\{y_{k}(a+k d) \mid y_{k} \in\{1, \ldots, q-1\}\right\} \\
& \bigcup\left\{\begin{array}{l|l}
(a+i d)+y_{k}(a+k d) & \begin{array}{l}
y_{k} \in\{0, \ldots, q-1\} \\
i \in\{1, \ldots, k-1\}
\end{array}
\end{array}\right\}
\end{aligned}
$$


Case b) If $r=1$ then

$$
\begin{aligned}
& A p(S, a) \backslash\{0\}=\left\{y_{k}(a+k d) \mid y_{k} \in\{1, \ldots, q\}\right\} \\
& \bigcup\left\{\begin{array}{l|l}
(a+i d)+y_{k}(a+k d) \mid \begin{array}{l}
y_{k} \in\{0, \ldots, q-1\} \\
i \in\{r, \ldots, k-1\}
\end{array}
\end{array}\right\}
\end{aligned}
$$

Case c) If $r \geq 2$ then

$$
\begin{aligned}
& A p(S, a) \backslash\{0\}=\left\{y_{k}(a+k d) \mid y_{k} \in\{1, \ldots, q\}\right\} \\
& \left.\begin{array}{l}
\bigcup\left\{\begin{array}{l|l}
(a+i d)+y_{k}(a+k d) & \begin{array}{l}
y_{k} \in\{0, \ldots, q\} \\
i \in\{1, \ldots, r-1\}
\end{array} \\
y_{k} \in\{0, \ldots, q-1\} \\
i \in\{r, \ldots, k-1\}
\end{array}\right.
\end{array}\right\}
\end{aligned}
$$

Suppose that $r=0$, i.e. $a=q k$. For $x \in \mathbb{Z} \backslash\{0, a\}$, we have

$$
\mu_{S}(x)=-\sum_{y_{k}=1}^{q-1} \mu_{S}\left(x-y_{k}(a+k d)\right)-\sum_{i=1}^{k-1} \sum_{y_{k}=0}^{q-1} \mu_{S}\left(x-(a+i d)-y_{k}(a+k d)\right) .
$$

By applying (10) to $x-(a+k d) \in \mathbb{Z} \backslash\{0, a\}$, that is, $x \in \mathbb{Z} \backslash\{a+k d, a+(a+k d)\}$ we obtain

$$
\mu_{S}(x-(a+k d))=-\sum_{y_{k}=2}^{q} \mu_{S}\left(x-y_{k}(a+k d)\right)-\sum_{i=1}^{k-1} \sum_{y_{k}=1}^{q} \mu_{S}\left(x-(a+i d)-y_{k}(a+k d)\right) .
$$

By combining (10) and (11) for $x \in \mathbb{Z} \backslash\{0, a, a+k d, a+(a+k d)\}$, we obtain

$$
\mu_{S}(x)=\mu_{S}(x-q(a+k d))+\sum_{i=1}^{k-1} \mu_{S}(x-(a+i d)-q(a+k d))-\mu_{S}(x-(a+i d)) .
$$

The cases when $r \geq 1$ are similar to Case a (and it is left to the reader as an exercise).

$$
\text { 6. CASE }\langle 2 q, 2 q+d, 2 q+2 d\rangle
$$

The multiplicity function of a multiset $A$ of $\mathbb{N}$ is the function

$$
\mathfrak{m}_{A}: \mathbb{N} \longrightarrow \mathbb{N}
$$

which assigns to each element $x \in \mathbb{N}$ its multiplicity, that is, the number of times that $x$ appears in the multiset $A$.

Let $a=2 q$ and $d \in \mathbb{N}^{*}$ such that $\operatorname{gcd}(a, d)=\operatorname{gcd}(q, d)=1$. For each $i \in\{-1,0,1\}$, we consider the following multisets.

$$
\begin{aligned}
A_{i} & =\{m(q+d)+i \mid m \in \mathbb{N}\}, \\
B_{i} & =\{m(q+d)-n d+i \mid m \in \mathbb{N} \backslash\{0,1\}, n \in\{1,2, \ldots,\lfloor m / 2\rfloor\}\}, \\
C_{i} & =A_{i} \bigcup B_{i} .
\end{aligned}
$$

As we mentioned above, given a triple $\left(x_{0}, x_{1}, x_{2}\right) \in \mathbb{N} \times\{0,1\} \times\{0, \ldots, q-1\}$, we denote by $\left[x_{0}, x_{1}, x_{2}\right]$ the element in $S$ given by the representation in Lemma 1 . In the sequel of this section, we shall consider this representation for all $x_{0} \in \mathbb{Z}$, i.e.,

$$
\left[x_{0}, x_{1}, x_{2}\right]=x_{0} a+x_{1}(a+d)+x_{2}(a+2 d)
$$


for all $\left(x_{0}, x_{1}, x_{2}\right) \in \mathbb{Z} \times\{0,1\} \times\{0, \ldots, q-1\}$. In this case, it is clear that

$$
\text { if }\left(x_{0}, x_{1}, x_{2}\right) \in \mathbb{Z} \times\{0,1\} \times\{0, \ldots, q-1\} \text { then }\left[x_{0}, x_{1}, x_{2}\right] \in S \Longleftrightarrow x_{0} \in \mathbb{N} \text {. }
$$

The latter will be used in the proofs below.

Theorem 3. Let $S=<2 q, 2 q+d, 2 q+2 d>$. Let $\left(x_{0}, x_{1}, x_{2}\right) \in \mathbb{Z} \times\{0,1\} \times\{0, \ldots, q-1\}$. Then,

$$
\mu_{S}\left(\left[x_{0}, x_{1}, x_{2}\right]\right)= \begin{cases}(-1)^{x_{1}}\left(\mathfrak{m}_{A_{0}}-\mathfrak{m}_{A_{1}}+2 \mathfrak{m}_{B_{0}}-\mathfrak{m}_{B_{-1}}-\mathfrak{m}_{B_{1}}\right)\left(x_{0}\right) & \text { if } x_{2}=0 \\ (-1)^{x_{1}}\left(2 \mathfrak{m}_{C_{0}}-\mathfrak{m}_{C_{-1}}-\mathfrak{m}_{C_{1}}\right)\left(x_{0}-x_{2}\right) & \text { if } x_{2} \geq 1 .\end{cases}
$$

We notice that if $x_{0}-x_{2}$ is a constant then we should have the same value for $\mu_{S}\left(\left[x_{0}, 0, x_{2}\right]\right)$. The latter is illustrated by the first values of $\mu_{S}\left(\left[x_{0}, 0, x_{2}\right]\right)$, listed in Table 1 given at the end of the section, for the case when $a=22$ and $d=5$. Indeed, we can see appearing diagonals (corresponding to $x_{0}-x_{2}$ constant) with the same value.

Before proving Theorem 3, we need two lemmas and the following refinement of Theorem 2 when $k=2$ and $a$ even.

Proposition 2. Let $\left(x_{0}, x_{1}, x_{2}\right) \in \mathbb{Z} \times\{0,1\} \times\{0, \ldots, q-1\}$ with $\left(x_{0}, x_{2}\right) \notin\{0,1\} \times\{0,1\}$. Then,

$$
\begin{aligned}
\mu_{S}\left(\left[x_{0}, x_{1}, 0\right]\right)= & \mu_{S}\left(\left[x_{0}-(q+d), x_{1}, 0\right]\right) \\
& +\mu_{S}\left(\left[x_{0}-(q+d)-1, x_{1}, q-1\right]\right) \\
& -\mu_{S}\left(\left[x_{0}-2(q+d)-1, x_{1}, q-1\right]\right)
\end{aligned}
$$

and

$$
\begin{aligned}
\mu_{S}\left(\left[x_{0}, x_{1}, x_{2}\right]\right)= & \mu_{S}\left(\left[x_{0}-(q+d), x_{1}, x_{2}\right]\right) \\
& +\mu_{S}\left(\left[x_{0}-1, x_{1}, x_{2}-1\right]\right) \\
& -\mu_{S}\left(\left[x_{0}-(q+d)-1, x_{1}, x_{2}-1\right]\right)
\end{aligned}
$$

when $x_{2} \geq 1$.

Proof. From Theorem 2, we have

$$
\begin{aligned}
\mu_{S}\left(\left[x_{0}, x_{1}, x_{2}\right]\right)= & \mu_{S}\left(\left[x_{0}, x_{1}, x_{2}\right]-[q+d, 0,0]\right)+\mu_{S}\left(\left[x_{0}, x_{1}, x_{2}\right]-[q+d, 1,0]\right) \\
& -\mu_{S}\left(\left[x_{0}, x_{1}, x_{2}\right]-[0,1,0]\right) .
\end{aligned}
$$

Notice that $q(a+2 d)=(q+d) a$ and $a+(a+2 d)=2(a+d)$.

Case a) If $x_{1}=0$ and $x_{2}=0$ then, from (12) we obtain

$$
\begin{aligned}
\mu_{S}\left(\left[x_{0}, 0,0\right]\right)= & \mu_{S}\left(\left[x_{0}, 0,0\right]-[q+d, 0,0]\right)+\mu_{S}\left(\left[x_{0}, 0,0\right]-[q+d, 1,0]\right) \\
& -\mu_{S}\left(\left[x_{0}, 0,0\right]-[0,1,0]\right) . \\
= & \mu_{S}\left(\left[x_{0}-(q+d), 0,0\right]\right)+\mu_{S}\left(\left[x_{0}-2(q+d)-1,1, q-1\right]\right) \\
& -\mu_{S}\left(\left[x_{0}-(q+d)-1,1, q-1\right]\right) .
\end{aligned}
$$


By applying the recursive equality (12) to $\mu_{S}\left(\left[x_{0}-(q+d)-1,1, q-1\right]\right)$, we obtain

$$
\begin{aligned}
\mu_{S}\left(\left[x_{0}-(q+d)-1,1, q-1\right]\right)= & \mu_{S}\left(\left[x_{0}-2(q+d)-1,1, q-1\right]\right) \\
& +\mu_{S}\left(\left[x_{0}-2(q+d)-1,0, q-1\right]\right) \\
& -\mu_{S}\left(\left[x_{0}-(q+d)-1,0, q-1\right]\right) .
\end{aligned}
$$

Finally, by combining equations (13) and (14) we have

$$
\begin{aligned}
\mu_{S}\left(\left[x_{0}, 0,0\right]\right)= & \mu_{S}\left(\left[x_{0}-(q+d), 0,0\right]\right) \\
& +\mu_{S}\left(\left[x_{0}-(q+d)-1,0, q-1\right]\right) \\
& -\mu_{S}\left(\left[x_{0}-2(q+d)-1,0, q-1\right]\right) .
\end{aligned}
$$

Case b) If $x_{1}=0$ and $x_{2} \geq 1$ then, from (12) we obtain

$$
\begin{aligned}
\mu_{S}\left(\left[x_{0}, 0, x_{2}\right]\right)= & \mu_{S}\left(\left[x_{0}, 0, x_{2}\right]-x[q+d, 0,0]\right)+\mu_{S}\left(\left[x_{0}, 0, x_{2}\right]-[q+d, 1,0]\right) \\
& -\mu_{S}\left(\left[x_{0}, 0, x_{2}\right]-x[0,1,0]\right) \\
= & \mu_{S}\left(\left[x_{0}-(q+d), 0, x_{2}\right]\right)+\mu_{S}\left(\left[x_{0}-(q+d)-1,1, x_{2}-1\right]\right) \\
& -\mu_{S}\left(\left[x_{0}-1,1, x_{2}-1\right]\right) .
\end{aligned}
$$

By applying the recursive equality (12) to $\mu_{S}\left(\left[x_{0}-1,1, x_{2}-1\right]\right)$, we obtain

$$
\begin{aligned}
\mu_{S}\left(\left[x_{0}-1,1, x_{2}-1\right]\right)= & \mu_{S}\left(\left[x_{0}-(q+d)-1,1, x_{2}-1\right]\right) \\
& +\mu_{S}\left(\left[x_{0}-(q+d)-1,0, x_{2}-1\right]\right) \\
& -\mu_{S}\left(\left[x_{0}-1,0, x_{2}-1\right]\right) .
\end{aligned}
$$

Finally, by combining equations (14) and (16) we have

$$
\begin{aligned}
\mu_{S}\left(\left[x_{0}, 0, x_{2}\right]\right)= & \mu_{S}\left(\left[x_{0}-(q+d), 0, x_{2}\right]\right) \\
& +\mu_{S}\left(\left[x_{0}-1,0, x_{2}-1\right]\right) \\
& -\mu_{S}\left(\left[x_{0}-(q+d)-1,0, x_{2}-1\right]\right) .
\end{aligned}
$$

This concludes the proof for $x_{1}=0$. The proof for the case $x_{1}=1$ is similar as the above case and it is left to the reader.

Lemma 2. Let $i \in\{-1,0,1\}$. For all $x \in \mathbb{Z} \backslash\{i\}, \mathfrak{m}_{A_{i}}(x)=\mathfrak{m}_{A_{i}}(x-(q+d))$.

Proof. By definition of the sets $A_{i}$, for any integer $x \leq q+d+i-1$ such that $x \neq i$, we have

$$
\mathfrak{m}_{A_{i}}(x)=\mathfrak{m}_{A_{i}}(x-(q+d))=0 .
$$

For any integer $x \geq q+d+i$, we obtain

$$
\begin{aligned}
x \in A_{i} & \Longleftrightarrow \text { there exists } m \in \mathbb{N} \text { with } x=m(q+d)+i \\
& \Longleftrightarrow \text { there exists } m \in \mathbb{N} \text { with } x-(q+d)=(m-1)(q+d)+i \\
& \Longleftrightarrow x-(q+d) \in A_{i} .
\end{aligned}
$$

This completes the proof.

Lemma 3. Let $i \in\{-1,0,1\}$. For all $x \in \mathbb{Z}, \mathfrak{m}_{B_{i}}(x)=\mathfrak{m}_{C_{i}}(x-(2 q+d))$. 
Proof. By definition of the multisets $B_{i}$ and $C_{i}$, for any integer $x \leq 2 q+d+i-1$, we have

$$
\mathfrak{m}_{B_{i}}(x)=\mathfrak{m}_{C_{i}}(x-(2 q+d))=0 .
$$

For any integer $x \geq 2 q+d+i$, we obtain

$$
\begin{aligned}
x \in B_{i} & \Longleftrightarrow \text { there exists } m \in \mathbb{N} \backslash\{0,1\}, 1 \leq n \leq\lfloor m / 2\rfloor \text { with } x=m(q+d)-n d+i \\
& \Longleftrightarrow \text { there exists } m \in \mathbb{N} \backslash\{0,1\}, 1 \leq n \leq\lfloor m / 2\rfloor \text { with } x-(2 q+d)=(m-2)(q+d) \\
& \Longleftrightarrow \text { there exists } m \in \mathbb{N}, 0 \leq n \leq\lfloor m / 2\rfloor \text { with } x-(2 q+d)=m(q+d)-n d+i \\
& \Longleftrightarrow x-(2 q+d) \in C_{i} .
\end{aligned}
$$

This completes the proof.

We may now prove Theorem 3.

Proof of Theorem 3. By double induction on $x_{0}$ and $x_{2}$.

For $x_{0}<0$, since $\left[x_{0}, x_{1}, x_{2}\right] \notin S$ and $C_{i} \cap(\mathbb{Z} \backslash \mathbb{N})=\emptyset$ for all $i \in\{-1,0,1\}$, it follows that

$$
\mu_{S}\left(\left[x_{0}, x_{1}, 0\right]\right)=(-1)^{x_{1}}\left(\mathfrak{m}_{A_{0}}-\mathfrak{m}_{A_{1}}+2 \mathfrak{m}_{B_{0}}-\mathfrak{m}_{B_{-1}}-\mathfrak{m}_{B_{1}}\right)\left(x_{0}\right)=0,
$$

and

$$
\mu_{S}\left(\left[x_{0}, x_{1}, x_{2}\right]\right)=(-1)^{x_{1}}\left(2 \mathfrak{m}_{C_{0}}-\mathfrak{m}_{C_{-1}}-\mathfrak{m}_{C_{1}}\right)\left(x_{0}-x_{2}\right)=0,
$$

for all $x_{2} \in\{1, \ldots, q-1\}$.

Now, for $x_{0} \in \mathbb{N}$, we suppose that the theorem is true for all values lesser than $x_{0}$ and all $x_{2} \in\{0, \ldots, q-1\}$. We distinguish different cases according to the values of $x_{2}$.

Case a) $x_{2}=0$.

For $x_{0}=0$, since $\mu_{S}([0,0,0])=\mu_{S}(0)=1$ and $\mu_{S}([0,1,0])=\mu_{S}(a+d)=-1$, it follows, by definition of the multisets $A_{i}$ and $B_{i}$, that

$$
\mu_{S}\left(\left[0, x_{1}, 0\right]\right)=(-1)^{x_{1}}=(-1)^{x_{1}}\left(\mathfrak{m}_{A_{0}}-\mathfrak{m}_{A_{1}}+2 \mathfrak{m}_{B_{0}}-\mathfrak{m}_{B_{-1}}-\mathfrak{m}_{B_{1}}\right)(0) .
$$

For $x_{0}=1$, since $\mu_{S}([1,0,0])=\mu_{S}(a)=-1$ and $\mu_{S}([1,1,0])=\mu_{S}(a+(a+d))=c_{2}(0,2 a+d)-$ $c_{1}(0,2 a+d)=2-1=1$, it follows, by definition of the multisets $A_{i}$ and $B_{i}$, that

$$
\mu_{S}\left(\left[1, x_{1}, 0\right]\right)=(-1)^{x_{1}+1}=(-1)^{x_{1}}\left(\mathfrak{m}_{A_{0}}-\mathfrak{m}_{A_{1}}+2 \mathfrak{m}_{B_{0}}-\mathfrak{m}_{B_{-1}}-\mathfrak{m}_{B_{1}}\right)(1) .
$$

Suppose now that $x_{0} \geq 2$. From Proposition 2, we have

$\mu_{S}\left(\left[x_{0}, x_{1}, 0\right]\right)=\mu_{S}\left(\left[x_{0}-(q+d), x_{1}, 0\right]\right)+\mu_{S}\left(\left[x_{0}-(q+d)-1, x_{1}, q-1\right]\right)-\mu_{S}\left(\left(x_{0}-2(q+d), x_{1}, q-1\right]\right)$.

By induction hypothesis, we have

$$
\begin{gathered}
\mu_{S}\left(\left[x_{0}-(q+d), x_{1}, 0\right]\right)=(-1)^{x_{1}}\left(\mathfrak{m}_{A_{0}}-\mathfrak{m}_{A_{1}}+2 \mathfrak{m}_{B_{0}}-\mathfrak{m}_{B_{-1}}-\mathfrak{m}_{B_{1}}\right)\left(x_{0}-(q+d)\right), \\
\mu_{S}\left(\left[x_{0}-(q+d)-1, x_{1}, q-1\right]\right)=(-1)^{x_{1}}\left(2 \mathfrak{m}_{C_{0}}-\mathfrak{m}_{C_{-1}}-\mathfrak{m}_{C_{1}}\right)\left(x_{0}-(2 q+d)\right)
\end{gathered}
$$

and

$$
\mu_{S}\left(\left[x_{0}-2(q+d)-1, x_{1}, q-1\right]\right)=(-1)^{x_{1}}\left(2 \mathfrak{m}_{C_{0}}-\mathfrak{m}_{C_{-1}}-\mathfrak{m}_{C_{1}}\right)\left(x_{0}-(3 q+2 d)\right) .
$$

By Lemma 2 , since $x_{0} \geq 2$, we already know that

$$
\mathfrak{m}_{A_{0}}\left(x_{0}-(q+d)\right)=\mathfrak{m}_{A_{0}}\left(x_{0}\right) \quad \text { and } \quad \mathfrak{m}_{A_{1}}\left(x_{0}-(q+d)\right)=\mathfrak{m}_{A_{1}}\left(x_{0}\right) .
$$

Moreover, by Lemma 3, we have

$$
\mathfrak{m}_{C_{i}}\left(x_{0}-(2 q+d)\right)=\mathfrak{m}_{B_{i}}\left(x_{0}\right) \quad \text { and } \quad \mathfrak{m}_{C_{i}}\left(x_{0}-(3 q+2 d)\right)=\mathfrak{m}_{B_{i}}\left(x_{0}-(q+d)\right),
$$


for all $i \in\{-1,0,1\}$. Therefore,

$$
\begin{aligned}
& \mu_{S}\left(\left[x_{0}, x_{1}, 0\right]\right)=(-1)^{x_{1}} {\left[\left(\mathfrak{m}_{A_{0}}-\mathfrak{m}_{A_{1}}\right)\left(x_{0}-(q+d)\right)\right.} \\
&+\left(2 \mathfrak{m}_{B_{0}}-\mathfrak{m}_{B_{-1}}-\mathfrak{m}_{B_{1}}\right)\left(x_{0}-(q+d)\right) \\
&+\left(2 \mathfrak{m}_{C_{0}}-\mathfrak{m}_{C_{-1}}-\mathfrak{m}_{C_{1}}\right)\left(x_{0}-(2 q+d)\right) \\
&\left.-\left(2 \mathfrak{m}_{C_{0}}-\mathfrak{m}_{C_{-1}}-\mathfrak{m}_{C_{1}}\right)\left(x_{0}-(3 q+2 d)\right)\right] \\
&=(-1)^{x_{1}} {\left[\left(\mathfrak{m}_{A_{0}}-\mathfrak{m}_{A_{1}}\right)\left(x_{0}\right)\right.} \\
&+\left(2 \mathfrak{m}_{B_{0}}-\mathfrak{m}_{B_{-1}}-\mathfrak{m}_{B_{1}}\right)\left(x_{0}-(q+d)\right) \\
&+\left(2 \mathfrak{m}_{B_{0}}-\mathfrak{m}_{B_{-1}}-\mathfrak{m}_{B_{1}}\right)\left(x_{0}\right) \\
&\left.-\left(2 \mathfrak{m}_{B_{0}}-\mathfrak{m}_{B_{-1}}-\mathfrak{m}_{B_{1}}\right)\left(x_{0}-(q+d)\right)\right] \\
&=(-1)^{x_{1}}\left(\mathfrak{m}_{A_{0}}-\mathfrak{m}_{A_{1}}+2 \mathfrak{m}_{B_{0}}-\mathfrak{m}_{B_{-1}}-\mathfrak{m}_{B_{1}}\right)\left(x_{0}\right) .
\end{aligned}
$$

Case b) $x_{2}=1$.

For $x_{0}=0$, since $\mu_{S}([0,0,1])=\mu_{S}(a+2 d)=-1$ and $\mu_{S}([0,1,1])=\mu_{S}((a+d)+(a+2 d))=$ $c_{2}(0,2 a+3 d)-c_{1}(0,2 a+3 d)=2-1=1$, it follows, by definition of the multisets $C_{i}$, that

$$
\mu_{S}\left(\left[0, x_{1}, 1\right]\right)=(-1)^{x_{1}+1}=(-1)^{x_{1}}\left(2 \mathfrak{m}_{C_{0}}-\mathfrak{m}_{C_{-1}}-\mathfrak{m}_{C_{1}}\right)(-1) .
$$

For $x_{0}=1$, since $\mu_{S}([1,0,1])=\mu_{S}(a+(a+2 d))=c_{2}(0,2 a+2 d)-c_{1}(0,2 a+2 d)=3-1=2$ and $\mu_{S}([1,1,1])=\mu_{S}(a+(a+d)+(a+2 d))=-c_{3}(0,3 a+3 d)+c_{2}(0,3 a+3 d)-c_{1}(0,3 a+3 d)=$ $-7+10-1=2$, it follows, by definition of the multisets $C_{i}$, that

$$
\mu_{S}\left(\left[1, x_{1}, 1\right]\right)=(-1)^{x_{1}} 2=(-1)^{x_{1}}\left(2 \mathfrak{m}_{C_{0}}-\mathfrak{m}_{C_{-1}}-\mathfrak{m}_{C_{1}}\right)(0) .
$$

Suppose now that $x_{0} \geq 2$. From Proposition 2, we have

$$
\mu_{S}\left(\left[x_{0},+x_{1}, 1\right]\right)=\mu_{S}\left(\left[x_{0}-(q+d), x_{1}, 1\right]\right)+\mu_{S}\left(\left[x_{0}-1, x_{1}, 0\right]\right)-\mu_{S}\left(\left[x_{0}-(q+d)-1, x_{1}, 0\right]\right) .
$$

By using Lemmas 2 and 3 and the induction hypothesis, we have

$$
\begin{gathered}
\mu_{S}\left(\left[x_{0}-(q+d), x_{1}, 1\right]\right)=(-1)^{x_{1}}\left(2 \mathfrak{m}_{C_{0}}-\mathfrak{m}_{C_{-1}}-\mathfrak{m}_{C_{1}}\right)\left(x_{0}-(q+d)-1\right), \\
\mu_{S}\left(\left[x_{0}-1, x_{1}, 0\right]\right)=(-1)^{x_{1}}\left(\mathfrak{m}_{A_{0}}-\mathfrak{m}_{A_{1}}+2 \mathfrak{m}_{B_{0}}-\mathfrak{m}_{B_{-1}}-\mathfrak{m}_{B_{1}}\right)\left(x_{0}-1\right)
\end{gathered}
$$

and

$$
\mu_{S}\left(\left[x_{0}-(q+d)-1, x_{1}, 0\right)=(-1)^{x_{1}}\left(\mathfrak{m}_{A_{0}}-\mathfrak{m}_{A_{1}}+2 \mathfrak{m}_{B_{0}}-\mathfrak{m}_{B_{-1}}-\mathfrak{m}_{B_{1}}\right)\left(x_{0}-(q+d)-1\right) .\right.
$$

First, since the multiset difference $C_{i} \backslash B_{i}$ is equal to $A_{i}$ for all $i \in\{-1,0,1\}$, it follows that

$$
\mathfrak{m}_{C_{i}}-\mathfrak{m}_{B_{i}}=\mathfrak{m}_{A_{i}}
$$

Therefore,

$$
\begin{aligned}
\mu_{S}\left(\left[x_{0}, x_{1}, 1\right]\right)=(-1)^{x_{1}} & {\left[\mathfrak{m}_{A_{0}}-\mathfrak{m}_{A_{1}}\right)\left(x_{0}-1\right)-\left(\mathfrak{m}_{A_{0}}-\mathfrak{m}_{A_{1}}\right)\left(x_{0}-(q+d)-1\right) } \\
& +\left(2 \mathfrak{m}_{C_{0}}-\mathfrak{m}_{C_{-1}}-\mathfrak{m}_{C_{1}}\right)\left(x_{0}-(q+d)-1\right) \\
& -\left(2 \mathfrak{m}_{B_{0}}-\mathfrak{m}_{B_{-1}}-\mathfrak{m}_{B_{1}}\right)\left(x_{0}-(q+d)-1\right) \\
& \left.+\left(2 \mathfrak{m}_{B_{0}}-\mathfrak{m}_{B_{-1}}-\mathfrak{m}_{B_{1}}\right)\left(x_{0}-1\right)\right] \\
=(-1)^{x_{1}} & {\left[\left(\mathfrak{m}_{A_{0}}-\mathfrak{m}_{A_{1}}\right)\left(x_{0}-1\right)-\left(\mathfrak{m}_{A_{0}}-\mathfrak{m}_{A_{1}}\right)\left(x_{0}-(q+d)-1\right)\right.} \\
& +\left(2 \mathfrak{m}_{A_{0}}-\mathfrak{m}_{A_{-1}}-\mathfrak{m}_{A_{1}}\right)\left(x_{0}-(q+d)-1\right) \\
& \left.+\left(2 \mathfrak{m}_{B_{0}}-\mathfrak{m}_{B_{-1}}-\mathfrak{m}_{B_{1}}\right)\left(x_{0}-1\right)\right] \\
=(-1)^{x_{1}} & {\left[\left(\mathfrak{m}_{A_{0}}-\mathfrak{m}_{A_{1}}\right)\left(x_{0}-1\right)+\left(\mathfrak{m}_{A_{0}}-\mathfrak{m}_{A_{-1}}\right)\left(x_{0}-(q+d)-1\right)\right.} \\
& \left.+\left(2 \mathfrak{m}_{B_{0}}-\mathfrak{m}_{B_{-1}}-\mathfrak{m}_{B_{1}}\right)\left(x_{0}-1\right)\right] . \\
& \quad 12
\end{aligned}
$$


Moreover, by Lemma 2 , since $x_{0} \geq 2$, we know that

$$
\mathfrak{m}_{A_{0}}\left(x_{0}-(q+d)-1\right)=\mathfrak{m}_{A_{0}}\left(x_{0}-1\right) \quad \text { and } \quad \mathfrak{m}_{A_{-1}}\left(x_{0}-(q+d)-1\right)=\mathfrak{m}_{A_{-1}}\left(x_{0}-1\right) .
$$

Finally, in this case, we obtain

$$
\begin{aligned}
\mu_{S}\left(\left[x_{0}, x_{1}, 1\right]\right)=(-1)^{x_{1}}\left[\left(\mathfrak{m}_{A_{0}}-\mathfrak{m}_{A_{1}}\right)\left(x_{0}-1\right)+\left(\mathfrak{m}_{A_{0}}-\mathfrak{m}_{A_{-1}}\right)\left(x_{0}-(q+d)-1\right)\right. \\
\left.\quad+\left(2 \mathfrak{m}_{B_{0}}-\mathfrak{m}_{B_{-1}}-\mathfrak{m}_{B_{1}}\right)\left(x_{0}-1\right)\right] . \\
=(-1)^{x_{1}}\left[\left(2 \mathfrak{m}_{A_{0}}-\mathfrak{m}_{A_{-1}}-\mathfrak{m}_{A_{1}}\right)\left(x_{0}-1\right)+\left(2 \mathfrak{m}_{B_{0}}-\mathfrak{m}_{B_{-1}}-\mathfrak{m}_{B_{1}}\right)\left(x_{0}-1\right)\right] \\
=(-1)^{x_{1}}\left(2 \mathfrak{m}_{C_{0}}-\mathfrak{m}_{C_{-1}}-\mathfrak{m}_{C_{1}}\right)\left(x_{0}-1\right) .
\end{aligned}
$$

Case c) $x_{2} \geq 2$.

From Proposition 2, we have

$\mu_{S}\left(\left[x_{0}, x_{1}, x_{2}\right]\right)=\mu_{S}\left(\left[x_{0}-(q+d), x_{1}, x_{2}\right]\right)+\mu_{S}\left(\left[x_{0}-1, x_{1}, x_{2}-1\right]\right)-\mu_{S}\left(\left[x_{0}-(q+d)-1, x_{1}, x_{2}-1\right]\right)$.

By induction, we have

$$
\begin{gathered}
\mu_{S}\left(\left[x_{0}-(q+d), x_{1} x_{2}\right]\right)=(-1)^{x_{1}}\left(2 \mathfrak{m}_{C_{0}}-\mathfrak{m}_{C_{-1}}-\mathfrak{m}_{C_{1}}\right)\left(x_{0}-x_{2}-(q+d)\right), \\
\mu_{S}\left(\left[x_{0}-1, x_{1}, x_{2}-1\right]\right)=(-1)^{x_{1}}\left(2 \mathfrak{m}_{C_{0}}-\mathfrak{m}_{C_{-1}}-\mathfrak{m}_{C_{1}}\right)\left(x_{0}-x_{2}\right)
\end{gathered}
$$

\begin{tabular}{|c|c|c|c|c|c|c|c|c|c|c|c|}
\hline$x_{2}$ & 0 & 1 & 2 & 3 & 4 & 5 & 6 & 7 & 8 & 9 & 10 \\
\hline 0 & 1 & -1 & 0 & 0 & 0 & 0 & 0 & 0 & 0 & 0 & 0 \\
\hline 1 & -1 & 2 & -1 & 0 & 0 & 0 & 0 & 0 & 0 & 0 & 0 \\
\hline 2 & 0 & -1 & 2 & -1 & 0 & 0 & 0 & 0 & 0 & 0 & 0 \\
\hline & 0 & 0 & -1 & 2 & -1 & 0 & 0 & 0 & 0 & 0 & 0 \\
\hline & 0 & 0 & 0 & -1 & 2 & -1 & 0 & 0 & 0 & 0 & 0 \\
\hline & 0 & 0 & 0 & 0 & -1 & 2 & -1 & 0 & 0 & 0 & 0 \\
\hline & 0 & 0 & 0 & 0 & 0 & -1 & 2 & -1 & 0 & 0 & 0 \\
\hline & 0 & 0 & 0 & 0 & 0 & 0 & -1 & 2 & -1 & 0 & 0 \\
\hline & 0 & 0 & 0 & 0 & 0 & 0 & 0 & -1 & 2 & -1 & 0 \\
\hline & 0 & 0 & 0 & 0 & 0 & 0 & 0 & 0 & -1 & 2 & -1 \\
\hline$q-1$ & 0 & 0 & 0 & 0 & 0 & 0 & 0 & 0 & 0 & -1 & 2 \\
\hline$q$ & 0 & 0 & 0 & 0 & 0 & 0 & 0 & 0 & 0 & 0 & -1 \\
\hline$q+1$ & 0 & 0 & 0 & 0 & 0 & 0 & 0 & 0 & 0 & 0 & 0 \\
\hline & 0 & 0 & 0 & 0 & 0 & 0 & 0 & 0 & 0 & 0 & 0 \\
\hline & 0 & 0 & 0 & 0 & 0 & 0 & 0 & 0 & 0 & 0 & 0 \\
\hline$q+d-1$ & 0 & 0 & 0 & 0 & 0 & 0 & 0 & 0 & 0 & 0 & 0 \\
\hline$q+d$ & 1 & -1 & 0 & 0 & 0 & 0 & 0 & 0 & 0 & 0 & 0 \\
\hline$q+d+1$ & -1 & 2 & -1 & 0 & 0 & 0 & 0 & 0 & 0 & 0 & 0 \\
\hline
\end{tabular}

and

$$
\mu_{S}\left(\left[x_{0}-(q+d)-1, x_{1}, x_{2}-1\right]\right)=(-1)^{x_{1}}\left(2 \mathfrak{m}_{C_{0}}-\mathfrak{m}_{C_{-1}}-\mathfrak{m}_{C_{1}}\right)\left(x_{0}-x_{2}-(q+d)\right) .
$$

Therefore,

$$
\begin{aligned}
\mu_{S}\left(\left[x_{0}, x_{1}, x_{2}\right]\right)=(-1)^{x_{1}} & {\left[\left(2 \mathfrak{m}_{C_{0}}-\mathfrak{m}_{C_{-1}}-\mathfrak{m}_{C_{1}}\right)\left(x_{0}-x_{2}-(q+d)\right)\right.} \\
& +\left(2 \mathfrak{m}_{C_{0}}-\mathfrak{m}_{C_{-1}}-\mathfrak{m}_{C_{1}}\right)\left(x_{0}-x_{2}\right) \\
& \left.-\left(2 \mathfrak{m}_{C_{0}}-\mathfrak{m}_{C_{-1}}-\mathfrak{m}_{C_{1}}\right)\left(x_{0}-x_{2}-(q+d)\right)\right] \\
=(-1)^{x_{1}} & \left(2 \mathfrak{m}_{C_{0}}-\mathfrak{m}_{C_{-1}}-\mathfrak{m}_{C_{1}}\right)\left(x_{0}-x_{2}\right) .
\end{aligned}
$$

This completes the proof of Theorem 3 .

Table 1: First values of $\mu_{S}\left(\left[x_{0}, 0, x_{2}\right]\right)$ for $q=11$ and $d=5$. 
Table 1 - continued from previous page

\begin{tabular}{|c|c|c|c|c|c|c|c|c|c|c|c|}
\hline$\succ_{x_{0}} \Upsilon^{x_{2}}$ & 0 & 1 & 2 & 3 & 4 & 5 & 6 & 7 & 8 & 9 & 10 \\
\hline \multirow[t]{8}{*}{$q+d+2$} & 0 & -1 & 2 & -1 & 0 & 0 & 0 & 0 & 0 & 0 & 0 \\
\hline & 0 & 0 & -1 & 2 & -1 & 0 & 0 & 0 & 0 & 0 & 0 \\
\hline & 0 & 0 & 0 & -1 & 2 & -1 & 0 & 0 & 0 & 0 & 0 \\
\hline & 0 & 0 & 0 & 0 & -1 & 2 & -1 & 0 & 0 & 0 & 0 \\
\hline & 0 & 0 & 0 & 0 & 0 & -1 & 2 & -1 & 0 & 0 & 0 \\
\hline & 0 & 0 & 0 & 0 & 0 & 0 & -1 & 2 & -1 & 0 & 0 \\
\hline & 0 & 0 & 0 & 0 & 0 & 0 & 0 & -1 & 2 & -1 & 0 \\
\hline & 0 & 0 & 0 & 0 & 0 & 0 & 0 & 0 & -1 & 2 & -1 \\
\hline $2 q+d-1$ & -1 & 0 & 0 & 0 & 0 & 0 & 0 & 0 & 0 & -1 & 2 \\
\hline $2 q+d$ & 2 & -1 & 0 & 0 & 0 & 0 & 0 & 0 & 0 & 0 & -1 \\
\hline \multirow[t]{3}{*}{$2 q+d+1$} & -1 & 2 & -1 & 0 & 0 & 0 & 0 & 0 & 0 & 0 & 0 \\
\hline & 0 & -1 & 2 & -1 & 0 & 0 & 0 & 0 & 0 & 0 & 0 \\
\hline & 0 & 0 & -1 & 2 & -1 & 0 & 0 & 0 & 0 & 0 & 0 \\
\hline $2 q+2 d-1$ & $\mathbf{0}$ & 0 & 0 & -1 & 2 & -1 & 0 & 0 & 0 & 0 & 0 \\
\hline $2 q+2 d$ & 1 & -1 & 0 & 0 & -1 & 2 & -1 & 0 & 0 & 0 & 0 \\
\hline $2 q+2 d+1$ & -1 & 2 & -1 & 0 & 0 & -1 & 2 & -1 & 0 & 0 & 0 \\
\hline \multirow[t]{8}{*}{$2 q+2 d+2$} & 0 & -1 & 2 & -1 & 0 & 0 & -1 & 2 & -1 & 0 & 0 \\
\hline & 0 & 0 & -1 & 2 & -1 & 0 & 0 & -1 & 2 & -1 & 0 \\
\hline & 0 & 0 & 0 & -1 & 2 & -1 & 0 & 0 & -1 & 2 & -1 \\
\hline & 0 & 0 & 0 & 0 & -1 & 2 & -1 & 0 & 0 & -1 & 2 \\
\hline & 0 & 0 & 0 & 0 & 0 & -1 & 2 & -1 & 0 & 0 & -1 \\
\hline & 0 & 0 & 0 & 0 & 0 & 0 & -1 & 2 & -1 & 0 & 0 \\
\hline & 0 & 0 & 0 & 0 & 0 & 0 & 0 & -1 & 2 & -1 & 0 \\
\hline & 0 & 0 & 0 & 0 & 0 & 0 & 0 & 0 & -1 & 2 & -1 \\
\hline $3 q+2 d-1$ & -1 & 0 & 0 & 0 & 0 & 0 & 0 & 0 & 0 & -1 & 2 \\
\hline $3 q+2 d$ & 2 & -1 & 0 & 0 & 0 & 0 & 0 & 0 & 0 & 0 & -1 \\
\hline \multirow[t]{3}{*}{$3 q+2 d+1$} & -1 & 2 & -1 & 0 & 0 & 0 & 0 & 0 & 0 & 0 & 0 \\
\hline & 0 & -1 & 2 & -1 & 0 & 0 & 0 & 0 & 0 & 0 & 0 \\
\hline & 0 & 0 & -1 & 2 & -1 & 0 & 0 & 0 & 0 & 0 & 0 \\
\hline $3 q+3 d-1$ & $\mathbf{0}$ & 0 & 0 & -1 & 2 & -1 & 0 & 0 & 0 & 0 & 0 \\
\hline $3 q+3 d$ & 1 & -1 & 0 & 0 & -1 & 2 & -1 & 0 & 0 & 0 & 0 \\
\hline $3 q+3 d+1$ & -1 & 2 & -1 & 0 & 0 & -1 & 2 & -1 & 0 & 0 & 0 \\
\hline \multirow[t]{3}{*}{$3 q+3 d+2$} & 0 & -1 & 2 & -1 & 0 & 0 & -1 & 2 & -1 & 0 & 0 \\
\hline & 0 & 0 & -1 & 2 & -1 & 0 & 0 & -1 & 2 & -1 & 0 \\
\hline & 0 & 0 & 0 & -1 & 2 & -1 & 0 & 0 & -1 & 2 & -1 \\
\hline
\end{tabular}

\section{REFERENCES}

[1] J.A. Deddens, A combinatorial identity involving relatively prime integers, J. Combin. Theory Ser. A 26 (1979) 189-192.

[2] J.L. Ramírez Alfonsín, The Diophantine Frobenius problem, Oxford Lecture Series in Mathematics and its Applications 30, Oxford University Press, Oxford, 2005.

[3] J.B. Roberts, Note on linear forms, Proc. Amer. Math. Soc. 7 (1956), 465-469.

[4] J.C. Rosales and P.A. García-Sánchez, Numerical Semigroups, Developments in Mathematics, 20, Springer, New-York, 2009.

[5] G.C. Rota, On the Foundations of Combinatorial Theory I. Theory of Möbius Functions, Z. Wahrscheinlichkeitstheorie 2 (1964) 340-368. 
Institut de Mathématiques et de Modélisation de Montpellier, Université Montpellier 2, Place EugÈne Bataillon, 34095 Montpellier

E-mail address: jonathan.chappelon@math.univ-montp2.fr

URL: http://www.math.univ-montp2.fr/ chappelon/

Institut de Mathématiques et de Modélisation de Montpellier, Université Montpellier 2, Place EugÈne Bataillon, 34095 Montpellier

E-mail address: jramirez@math.univ-montp2.fr

URL: http://www.math.univ-montp2.fr/〜ramirez/ 